\title{
Desenvolvimento e Implementação de um Registo de Doentes: Experiência de um Centro de Esclerose Múltipla em Portugal
}

\section{Development and Implementation of a Patient Registry: The Experience of a Multiple Sclerosis Center in Portugal}

\author{
João de SÁ ${ }^{1,2}$, João FERREIRA ${ }^{1}$, Ana MACEDO $\triangle^{3,4}$ \\ Acta Med Port 2022 May;35(5):328-335 - https://doi.org/10.20344/amp.13933
}

RESUMO

Registos de doentes permitem avaliar melhor resultados terapêuticos e suportar a personalização de cuidados de saúde em diversas patologias. Este trabalho teve como objetivos: implementar um registo local num centro de esclerose múltipla em Portugal; proceder a uma análise crítica das suas etapas de desenvolvimento; e realizar uma primeira análise dos doentes incluídos.

Material e Métodos: A criação do registo dividiu-se em duas fases: desenvolvimento (construção da plataforma online) e implementação (recrutamento de doentes e recolha retrospetiva e prospetiva da informação disponível). Realizou-se uma análise demográfica e clínica dos doentes incluídos.

Resultados: Especialistas em Neurologia e coordenadores de estudo participaram no projeto, num total de 1050 horas de trabalho na fase de implementação. Dos 498 doentes com esclerose múltipla incluídos no estudo, $72,9 \%$ eram do género feminino, tendo sido identificada como subtipo de doença mais comum a esclerose múltipla surto remissão. Os fármacos mais frequentemente prescritos após diagnóstico foram interferões beta. Detetaram-se lacunas no processo clínico dos doentes relativamente à progressão da incapacidade e a exames complementares de diagnóstico.

Conclusão: Apesar do volume de dados recolhidos no âmbito deste estudo, foram encontradas dificuldades que comprometeram a implementação e manutenção do registo, que poderão ser ultrapassadas com a otimização de estratégias futuras.

Palavras-chave: Esclerose Múltipla; Estudos Prospetivos; Estudos Retrospetivos; Processos Clínicos; Registos

\section{ABSTRACT}

Introduction: Patient registries allow better evaluations of therapeutic outcomes and support personalized health care in several conditions. This study aimed to implement a local registry in a multiple sclerosis center in Portugal, in order to carry out a critical analysis of its development stages, and to perform an initial analysis of the included patients.

Material and Methods: The establishment of the registry was divided in two phases - development (creation of the online platform for data entry) and implementation (recruitment of patients and retrospective and prospective collection of available information). A demographic and clinical analysis of patients was performed.

Results: Neurologists and study coordinators participated in the project, accounting for a total of 1050 hours of work in the implementation phase. Amongst the 498 multiple sclerosis patients included, $72.9 \%$ were female and relapsing-remitting multiple sclerosis was the most common subtype of the disease. The most frequently prescribed drugs at diagnosis were beta interferons. Missing data in electronic health records were detected concerning the progression of disability and diagnostic tests.

Conclusion: Despite the amount of data collected within the scope of this study, several difficulties affected the implementation and maintenance of the registry, which could be overcome by improving future strategies.

Keywords: Medical Records; Multiple Sclerosis; Prospective Studies; Registries; Retrospective Studies

\section{INTRODUÇÃO}

Os registos de doentes são muito mais que bases de dados; são programas organizados e sistemáticos de compilação, armazenamento e divulgação de dados de indivíduos, identificados para um propósito específico e claramente explicitado junto dos mesmos. ${ }^{1}$

A integração de registos e bases de dados com elevado nível de qualidade em sistemas abrangentes de gestão de qualidade em Saúde, é um factor de melhoria das práticas clínicas, e dos resultados de saúde obtidos. ${ }^{2}$

A nível global, os registos de doentes de âmbito nacio- nal permitem a melhor avaliação dos resultados terapêuticos pelos clínicos, fornecendo informação mais detalhada e abrangente do que pequenos estudos locais. ${ }^{3}$ No entanto, é de realçar que a criação de sistemas de registo funcionais e com utilidade assegurada é um processo gradual que requer tempo, estudos prévios e acumulação de resultados. ${ }^{4}$

A esclerose múltipla (EM) é uma patologia neurológica heterogénea, com considerável variação inter-individual em termos de fenótipos clínicos, achados laboratoriais e imagiológicos, curso de doença e resposta à terapêutica.

\footnotetext{
1. Serviço de Neurologia. Hospital de Santa Maria. Centro Hospitalar Universitário de Lisboa Norte. Lisboa. Portugal.

2. Departamento de Neurologia. Faculdade de Medicina. Universidade de Lisboa. Lisboa. Portugal.

3. Faculdade de Medicina e Ciências Biomédicas. Universidade do Algarve. Faro. Portugal.

4. Keypoint. Grupo Evidenze. Lisboa. Portugal.

$\square$ Autor correspondente: Ana Macedo. amacedo@keypoint.pt

Recebido/Received: 16/04/2020 - Aceite/Accepted: 10/08/2020 - Publicado Online/Published Online: 18/01/2021 - Publicado/Published: 02/05/2022 Copyright $\odot$ Ordem dos Médicos 2022
} 
Estas características levam a quese adote uma abordagem multifatorial na personalização de cuidados aos doentes com EM que é hoje preconizada, com análise de múltiplos parâmetros. ${ }^{5}$

Os registos de doentes permitem a captura de dados transversais e longitudinais que seriam de outra forma inacessíveis. ${ }^{6}$ No caso da EM, apresentam precisamente a mais-valia de poderem suportar a personalização de cuidados de saúde aos doentes com esta patologia. ${ }^{7}$

Neste contexto, definimos como objetivo deste trabalho a criação e manutenção de um registo local num centro de EM em Portugal. Complementarmente, propusemo-nos proceder a uma análise crítica das suas etapas de implementação e desenvolvimento. Finalmente, foi ainda objetivo deste estudo proceder à descrição demográfica e clínica dos doentes de EM incluídos no Registo, comparando-os com os doentes com primeira consulta mais recente no mesmo centro de EM, bem como com grupos de doentes descritos na literatura científica.

\section{MATERIAL E MÉTODOS}

Este registo local foi concebido como ferramenta de estudo observacional, longitudinal e unicêntrico. Como tal, foi avaliado e aprovado, em julho de 2017, pela Comissão de Ética do Centro Hospitalar de Lisboa Norte, EPE, e conduzido de acordo com as boas práticas clínicas e de investigação científica, bem como com a Declaração de Helsínquia.

\section{Desenvolvimento da plataforma de registo}

O Registo consiste numa plataforma online, cujo desenho foi amplamente discutido por médicos e informáticos de forma a construir uma plataforma alinhada com a informação recolhida em contexto de prática clínica, e que facilitasse o registo dos dados necessários, mas que, por outro lado, também permitisse a análise desses mesmo dados de forma direta e intuitiva. A fase de desenvolvimento incluiu a seleção de campos a preencher no Registo pelos autores do estudo, com base em conhecimento prévio de estudos na mesma área, e na organização dos processos clínicos do Hospital de Santa Maria (HSM). Esta fase incluiu ainda uma revisão, testes e validação final por parte dos médicos neurologistas autores do estudo (realizando, nomeadamente, testes de validação com base na recolha preliminar de dados de processos clínicos aleatórios), tendo sido realizadas as alterações necessárias, que corresponderam a três períodos de alterações na plataforma para uniformização e classificações.

Os campos de preenchimento da plataforma, organizados em 11 secções diferentes, podem ser consultados na Tabela 1. O user interface da plataforma foi desenvolvido em HTML/Javascript, a base de dados (BD) em MySQL e a interação com a BD foi programada em PHP.

$\mathrm{O}$ acesso à plataforma foi limitadoà equipa do centro, por recurso a password. A cada doente registado foi atribuído um código numérico sequencial, sem qualquer relação com o seu processo clínico do hospital, assim garantindo a
Tabela 1 - Variáveis a recolher no registo implementado

\begin{tabular}{|c|c|}
\hline Secção & Campo \\
\hline I. Caracterização & $\begin{array}{l}\text { No identificação do doente } \\
\text { Idade } \\
\text { Género } \\
\text { Escolaridade, situação profissional } \\
\text { Peso, altura }\end{array}$ \\
\hline II. Diagnóstico & $\begin{array}{l}\text { Data de diagnóstico de EM } \\
\text { - Sintomas } \\
\text { - Exames realizados (RM, PL, PE) } \\
\text { - EDSS } \\
\text { Data da primeira consulta no HSM } \\
\text { Data dos primeiros sintomas } \\
\text { - Sintomas } \\
\text { Subtipo de doença }\end{array}$ \\
\hline III. Registo de consultas & $\begin{array}{l}\text { Data da consulta } \\
\text { Estado clínico } \\
\text { Ocorrência de eventos adversos } \\
\text { Adesão à terapêutica } \\
\text { EDSS }\end{array}$ \\
\hline IV. Medicação & $\begin{array}{l}\text { Fármaco } \\
\text { Duração da terapêutica } \\
\text { Razão de descontinuação }\end{array}$ \\
\hline V. Doenças concomitantes & $\begin{array}{l}\text { Patologia } \\
\text { Data de diagnóstico }\end{array}$ \\
\hline VI. Intercorrências & $\begin{array}{l}\text { Diagnóstico } \\
\text { Duração }\end{array}$ \\
\hline VII. Gravidez & $\begin{array}{l}\text { Intenção de engravidar } \\
\text { Gravidezes anteriores }\end{array}$ \\
\hline VIII. Exames & $\begin{array}{l}\text { Data de realização } \\
\text { RM } \\
\text { - Tipo e número de lesões } \\
\text { - Agravamento } \\
\text { PL } \\
\text { - LCR } \\
\text { - igG } \\
\text { PE } \\
\text { - VEP, AEP, SEP }\end{array}$ \\
\hline IX. Surtos & $\begin{array}{l}\text { Data de ocorrência } \\
\text { Tipo de surto } \\
\text { Gravidade } \\
\text { Tratamento }\end{array}$ \\
\hline X. Análises & $\begin{array}{l}\text { Linfócitos } \\
\text { Leucócitos } \\
\text { Albumina } \\
\text { ALT, AST, ALP }\end{array}$ \\
\hline
\end{tabular}

EM: esclerose múltipla; RM: ressonância magnética; PL: punção lombar; PE: potenciais evocados visuais; EDSS: expanded disability status scale; HSM: Hospital de Santa Maria; LCR: líquido céfalo-raquidiano; igG: imunoglobulina G; VEP: potenciais evocados visuais; AEP: potenciais evocados auditivos; SEP: potenciais evocados somatossensitivos; ALT: alanina aminotransferase; AST: aspartato aminotransferase; ALP: fosfatase alcalina

anonimização dos dados.

No total, o processo de desenvolvimento teve uma duração de aproximadamente oito meses, e um número total de horas de trabalho estimado em 960 horas.

\section{Implementação do Registo}

A fase de implementação teve início em outubro de 2017, tendo o período de recrutamento de doentes sido de 
12 meses. Nesta primeira etapa, entre outubro de 2017 e outubro de 2018, foram convidados a participar no estudo todos os doentes seguidos na consulta de Neurologia do HSM com diagnóstico conhecido de EM, no decurso de uma consulta de rotina.

Foram utilizados como critérios de inclusão: doentes com diagnóstico de EM - definido como estando mencionado no processo clínico enquanto diagnóstico e utilizando a data de diagnóstico registada neste processo - junto dos quais fosse obtido consentimento informado escrito para recolha de informação do seu processo clínico no âmbito do presente estudo. Não foram considerados critérios de exclusão.

Todos os doentes que aceitaram participar assinaram um formulário de consentimento informado, que detalhava, de forma clara, a natureza e os objetivos de criação do Registo. Ainda nesta fase, após dado o consentimento por escrito, foi efetuada a recolha de alguns dados demográficos e clínicos do doente.

Para os doentes menores de idade (idade inferior a 18 anos) previu-se que seria(m) o(s) representante(s) legal(ais) a assinar os documentos, sendo que os doentes que durante o estudo atingissem a idade adulta (18 anos) teriam que assinar o respetivo consentimento informado nesse momento, e concordar com a utilização dos dados previamente recolhidos.

A segunda etapa de implementação do Registo consistiu na recolha sistemática e retrospetiva de dados clínicos, imagiológicos e laboratoriais dos doentes elegíveis que constassem do processo clínico no HSM, nomeadamente história clínica, primeiros sintomas, surtos, data de diagnóstico de EM, EDSS (expanded disability status scale), achados imagiológicos e resultados laboratoriais.
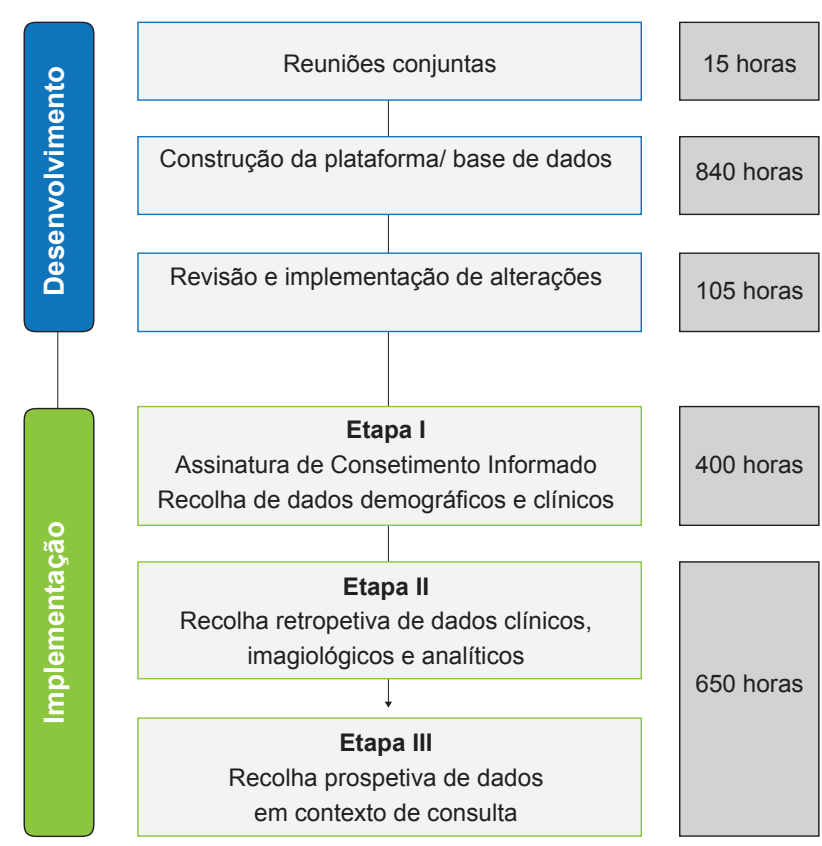

Figura 1 - Etapas de criação do registo implementado
A terceira e última etapa consistiu na recolha longitudinal prospetiva de dados clínicos, imagiológicos e laboratoriais dos doentes em contexto de consulta, em prática clínica habitual, especificamente o registo de ocorrência de surtos e a sua tipologia, dados laboratoriais com foco na contagem de linfócitos e nos indicadores de função hepática e ainda resultados de exames imagiológicos.

A segunda e terceira etapas decorreram entre março de 2018 e outubro de 2019.

\section{Caracterização dos doentes incluídos no Registo}

Procedeu-se a uma análise geral de caracterização demográfica e clínica de todos os doentes incluídos no Registo. Considerando-se que os doentes com primeira consulta entre 2016 e 2018 poderiam apresentar informação mais completa ou divergente nos registos em processo clínico, realizou-se ainda uma análise descritiva e comparativa deste subgrupo de doentes com o grupo geral de doentes.

\section{RESULTADOS}

\section{Análise de recursos para a fase de implementação e fase longitudinal do Registo}

Na fase de implementação, nomeadamente a etapa 1 (recrutamento), foram incluídos no Registo 498 doentes com EM acompanhados na consulta de Neurologia do HSM. Em termos de recursos humanos, participaram nesta fase os médicos da consulta de EM e os coordenadores de estudo afetos ao projeto. Estima-se que tenha sido gasto um total de 400 horas (150 horas por parte dos investigadores e 250 horas por parte dos coordenadores de estudo), o que corresponde a cerca de 36 horas por mês.

Em média, o tempo para apresentação do estudo e obtenção de consentimento informado foi de 10 minutos; o tempo relativo a questões diretas ao doente na avaliação inicial foi de sete minutos, e o tempo de revisão de processo e preenchimento da plataforma até finalização do registo individual foi de 15 minutos.

$\mathrm{Na}$ fase longitudinal, a de seguimento dos doentes registados, foram contabilizadas um total de 650 horas de trabalho, durante 20 meses. Estas incluem o tempo despendido pelo investigador no preenchimento dos dados em contexto de consulta, e a recolha de informação retrospetiva efetuada numa base regular de quatro a oito horas por semana.

As etapas de criação do Registo encontram-se resumidas na Fig. 1.

\section{Análise global de caracterização dos doentes incluídos no Registo \\ Foram incluídos no Registo 498 doentes com diagnós-} tico de EM. É importante realçar que se registaram muitas lacunas de informação nos dados recolhidos retrospetivamente a partir do processo clínico,

\section{Caracterização sociodemográfica}

Os doentes são maioritariamente do género feminino (72,9\%; $n=363$ ), com idade média de 45,8 anos (DP 11,9). 
Tabela 2 - Caracterização sociodemográfica dos doentes incluídos no registo implementado

\begin{tabular}{lcc}
\hline & Média & DP \\
\hline Idade atual, anos & 34,1 & 10,8 \\
IMC, $\mathrm{kg} / \mathrm{m}^{2}$ & 24,2 & 4,8 \\
\hline & $\mathbf{n}(\%)$ \\
\hline Género feminino & $363(72,9)$ \\
Escolaridade & $43(8,6)$ \\
$1^{\circ}$ ciclo do ensino básico & $70(14,1)$ \\
$2^{\circ} / 3^{\circ}$ ciclo do ensino básico & $160(32,1)$ \\
Ensino secundário & $172(34,5)$ \\
Licenciatura ou grau superior & $41(8,2)$ \\
Mestrado & $9(1,8)$ \\
Doutoramento & $3(0,6)$ \\
NS/NR & \\
Situação profissional & $268(53,8)$ \\
Ativo & $45(9,0)$ \\
Desempregado & $162(32,5)$ \\
Reformado & $17(3,4)$ \\
Estudante & $2(0,4)$ \\
Trabalhador estudante & $4(0,8)$ \\
Doméstico & \\
\hline DP: desvio padrão: NS/NR: Não sabe/ Não responde: IMC: indice de massa corporal
\end{tabular}

DP: desvio padrão; NS/NR: Não sabe/Não responde; IMC: índice de massa corporal

Do total de doentes registados, $45 \%(n=222)$ frequentou o ensino superior, e mais de metade encontrava-se profissionalmente ativo à data de inclusão $(53,8 \%$; $n=268)$. Adicionalmente, $32 \%$ dos doentes tinha excesso de peso (IMC > $25 \mathrm{~kg} / \mathrm{m}^{2}$ ) e $8 \%$ eram obesos (IMC > $30 \mathrm{~kg} / \mathrm{m}^{2}$ ) (Tabela 2).

\section{Caracterização da doença à data de diagnóstico}

No que diz respeito à caracterização clínica dos doentes, estes tinham entre seis e 70 anos de idade à data de diagnóstico (Média $=34,1 ; \mathrm{DP}=10,8$ ). O tempo mediano entre o diagnóstico e a primeira consulta no HSM foi de 31 meses, sendo que em $15 \%$ dos casos o diagnóstico foi efetuado na primeira consulta no HSM. As datas de diagnóstico registadas ocorreram entre 01 de junho de 1971 e 01 de dezembro de 2018.

O subtipo mais comum de EM foi esclerose múltipla surto remissão $(71,1 \%$ ) (Tabela 3$)$. O valor mediano de EDSS (expanded disability status scale) no diagnóstico era de 0 , com $29 \%$ dos doentes a revelar EDSS igual ou superior a 1 , e $19 \%$ igual ou superior a 2 . Esta variável encontrava-se registada somente em 195 dos doentes.

As manifestações clínicas mais comuns antes do diagnóstico foram alterações sensoriais $(45 \%)$, alterações da visão (36\%) e alterações motoras (25\%).

À data do diagnóstico, e de acordo com os registos nos processos clínicos, 33\% dos doentes tinham realizado ressonância magnética (RM), 20\% estudos de potenciais evocados e $15 \%$ punção lombar.

Os fármacos mais frequentemente prescritos no momento do diagnóstico foram interferão beta-1a (37\%), inter- ferão beta-1b (21\%) e acetato de glatirâmero (14\%).

\section{Evolução da doença e caracterização atual}

O tempo médio desde o diagnóstico dos doentes seguidos atualmente na consulta de EM do HSM é de 12,5 anos, e o tempo médio entre a primeira e a última consulta de cada doente é de 86 meses (tempo médio de seguimento). O número mediano de consultas é de 13 por doente, com número mediano anual de duas consultas (Tabela 4).

O número mediano de RM realizadas durante o seguimento foi de 1 , sendo a mediana por ano de 0,3 .

Durante o período de seguimento o número mediano de surtos foi de 1 , sendo o número mediano anualizado de 0,2 surtos por ano.

Os fármacos mais frequentes na última avaliação foram interferão beta-1a (16\%), fumarato de dimetilo (12\%) e acetato de glatirâmero (10\%).

\section{Caracterização do subgrupo de doentes com primeira consulta entre 2016 e 2018}

Cento e sete doentes foram avaliados na consulta de EM do HSM pela primeira vez entre janeiro de 2016 e dezembro de 2018.

Relativamente às características sociodemográficas deste subgrupo face à coorte total, destaca-se o seguinte: percentagem similar de mulheres $(68 \%$ doentes do sexo feminino); idade média inferior, 40,6 anos (DP 12,0), $p<$ 0,001; nível académico mais elevado (59\% com licenciatura ou grau académico superior, $p<0,001)$; percentagem de indivíduos profissionalmente ativos superior $(61,7 \%)$; e percentagem de doentes com excesso de peso ou obesidade similar (30\% e 5\%, respetivamente).

À data de diagnóstico, os doentes tinham entre 18 e 70 anos, média 36,2 (DP 11,8), similar aos valores da coorte total. O tempo mediano entre o diagnóstico e a primeira consulta no HSM foi de dois meses (inferior face à coorte total, $p<0,001$ ), sendo que em $26 \%$ dos casos o diagnóstico foi efetuado na primeira consulta no HSM.

O subtipo mais comum de EM foi esclerose múltipla surto remissão $(75,7 \%)$. O valor mediano de EDSS no diagnóstico era de 0 , com $21 \%$ dos doentes a revelar EDSS igual ou superior a 1, e $8 \%$ igual ou superior a 2. Mais uma vez, esta variável encontrava-se somente registada nos processos clínicos de 63 doentes.

As manifestações clínicas mais comuns antes do diagnóstico foram alterações sensoriais $(47 \%)$, alterações da visão (39\%) e alterações motoras (13\%).

À data de diagnóstico, $40 \%$ dos doentes tinham registo no processo clínico de realização de RM, $25 \%$ estudos de potenciais evocados, e $20 \%$ punção lombar.

Os fármacos mais frequentemente prescritos no momento do diagnóstico foram interferão beta-1a (35\%), fumarato de dimetilo (25\%), acetato de glatirâmero (17\%), e teriflunomida (16\%).

\section{DISCUSSÃO}

A criação de um Registo, independentemente da sua 
Tabela 3 - Caracterização da doença à data do diagnóstico

\begin{tabular}{|c|c|c|c|c|}
\hline & Média & DP & Mediana & $\| Q$ \\
\hline Idade à data do diagnóstico, anos & 34,1 & 10,8 & - & \\
\hline \multirow[t]{2}{*}{ Tempo entre diagnóstico e $1^{\text {a }}$ consulta no HSM, meses } & - & - & 31,0 & $2,0-104,5$ \\
\hline & \multicolumn{4}{|c|}{ n (\%) } \\
\hline \multicolumn{5}{|l|}{ Subtipo clínico de esclerose múltipla } \\
\hline EMSR & \multicolumn{4}{|c|}{$155(71,1)$} \\
\hline EMSP & \multicolumn{4}{|c|}{$33(15,1)$} \\
\hline EMPP & \multicolumn{4}{|c|}{$26(11,9)$} \\
\hline CIS & \multicolumn{4}{|c|}{$4(1,8)$} \\
\hline \multicolumn{5}{|l|}{ EDSS } \\
\hline$\geq 0$ & \multicolumn{4}{|c|}{$10(5,1)$} \\
\hline$\geq 1$ & \multicolumn{4}{|c|}{$56(28,7)$} \\
\hline$\geq 2$ & \multicolumn{4}{|c|}{$36(18,5)$} \\
\hline$\geq 3$ & \multicolumn{4}{|c|}{$19(9,7)$} \\
\hline$\geq 4$ & \multicolumn{4}{|c|}{$23(11,8)$} \\
\hline$\geq 5$ & \multicolumn{4}{|c|}{$26(13,3)$} \\
\hline $6-8$ & \multicolumn{4}{|c|}{$25(12,8)$} \\
\hline \multicolumn{5}{|l|}{ Manifestações clínicas } \\
\hline Alterações da visão & \multicolumn{4}{|c|}{$177(35,5)$} \\
\hline Alterações do tronco cerebral & \multicolumn{4}{|c|}{$16(3,2)$} \\
\hline Alterações motoras & \multicolumn{4}{|c|}{$122(24,5)$} \\
\hline Alterações do equilíbrio & \multicolumn{4}{|c|}{$67(13,5)$} \\
\hline Alterações sensoriais & \multicolumn{4}{|c|}{$224(45,0)$} \\
\hline Alterações do esfíncter & \multicolumn{4}{|c|}{$8(1,6)$} \\
\hline Alterações cognitivas & \multicolumn{4}{|c|}{$1(0,2)$} \\
\hline Fadiga & \multicolumn{4}{|c|}{$36(7,2)$} \\
\hline \multicolumn{5}{|l|}{ MCDTs } \\
\hline Ressonância magnética & \multicolumn{4}{|c|}{$166(33,3)$} \\
\hline Potenciais evocados & \multicolumn{4}{|c|}{$99(19,9)$} \\
\hline Punção lombar & & & & \\
\hline TMDs & & & & \\
\hline Interferão beta-1a & & & & \\
\hline Interferão beta-1b & & & & \\
\hline Peginterferão beta-1a & & & & \\
\hline Acetato de glatirâmero & & & & \\
\hline Teriflunomida & & & & \\
\hline Fingolimod & & & & \\
\hline Fumarato de dimetilo & & & & \\
\hline Natalizumab & & & & \\
\hline Cladribina & & & & \\
\hline
\end{tabular}

DP: desvio padrão; IIQ: intervalo inter-quartis; HSM: Hospital de Santa Maria; EDSS: expanded disability status scale; EMSR: esclerose múltipla surto remissão; EMSP: esclerose múltipla secundária progressiva; EMPP: esclerose múltipla primária progressiva; CIS: clinically isolated syndrome (síndrome clínica isolada); MCDTs: meios complementares de diagnóstico e terapêutica; TMDs: terapêuticas modificadoras da doença

abrangência clínica ou geográfica, implica procedimentos transversais, de que são exemplo a criação e desenvolvimento de plataformas e interface adequados, a inserção e validação de dados a implementação de processos de monitorização e qualidade. ${ }^{8}$

O desenvolvimento da plataforma que serviria de base ao Registo de EM implicou a articulação entre clínicos e outros profissionais do Serviço de Neurologia, assim como o contributo de um programador experiente. Esta dinâmica colaborativa permitiu que a plataforma final refletisse as necessidades e expectativas dos profissionais, previsivelmente potenciando a sua subsequente participação no 
Tabela 4 - Evolução da doença e caracterização atual dos doentes incluídos no registo implementado

\begin{tabular}{|c|c|c|c|c|}
\hline & Média & DP & Mediana & IIQ \\
\hline Tempo desde diagnóstico, anos & 12,5 & 9,0 & - & \\
\hline Tempo entre $1^{\text {a }}$ e última consulta no HSM, meses & 85,7 & 76,3 & - & \\
\hline $\mathrm{N}^{\circ}$ de consultas & & & 13,0 & $7,0-18,0$ \\
\hline $\mathrm{N}^{\circ}$ de consultas/ano & & & 2,0 & $1,5-2,7$ \\
\hline $\mathrm{N}^{\circ}$ de surtos & & & 1,0 & $1,0-2,0$ \\
\hline $\mathrm{N}^{\circ}$ de surtos/ano & & & 0,2 & $0,1-0,5$ \\
\hline $\mathrm{N}^{\circ}$ de $\mathrm{RM}$ & - & - & 1,0 & $1,0-2,0$ \\
\hline $\mathrm{N}^{\circ}$ de RM/ano & - & - & 0,3 & $0,1-0,5$ \\
\hline & \multicolumn{4}{|c|}{ n (\%) } \\
\hline \multicolumn{5}{|l|}{ TMDs } \\
\hline Interferão beta-1a & \multicolumn{4}{|c|}{$82(16,4)$} \\
\hline Interferão beta-1b & \multicolumn{4}{|c|}{$18(3,6)$} \\
\hline Peginterferão beta-1a & \multicolumn{4}{|c|}{$14(2,8)$} \\
\hline Acetato de glatirâmero & \multicolumn{4}{|c|}{$51(10,2)$} \\
\hline Teriflunomida & \multicolumn{4}{|c|}{$36(7,2)$} \\
\hline Fingolimod & \multicolumn{4}{|c|}{$43(8,6)$} \\
\hline Fumarato de dimetilo & \multicolumn{4}{|c|}{$58(11,6)$} \\
\hline Natalizumab & \multicolumn{4}{|c|}{$32(6,4)$} \\
\hline Cladribina & \multicolumn{4}{|c|}{$5(1,0)$} \\
\hline
\end{tabular}

DP: desvio padrão; IIQ: intervalo inter-quartis; TMDs: terapêuticas modificadoras da doença

adequado e completo preenchimento da plataforma.

Os resultados obtidos da análise de variáveis demográficas, designadamente a incidência da doença no género feminino, está de acordo com os dados epidemiológicos encontrados na literatura. ${ }^{9,10}$ Comparando os nossos resultados com investigação realizada em Portugal, os doentes incluídos no presente estudo são mais jovens e apresentam maior nível de escolaridade do que o descrito na literatura nacional. ${ }^{11}$ No que concerne às variáveis clínicas descritas, o subtipo esclerose múltipla surto remissão encontra-se descrito na literatura como o mais frequente, estando esta informação em concordância com os dados disponíveis da nossa amostra. ${ }^{12,13}$ Destacamos, porém, que os resultados obtidos $(71,1 \%)$ para a proporção deste subtipo de EM são superiores aos previamente publicados em Portugal. ${ }^{11}$

Comparativamente à coorte total, a população com primeira consulta entre 2016 e 2018 é mais jovem e diferenciada, e o tempo entre o diagnóstico e a primeira consulta no HSM é menor, o que indica uma melhoria nos cuidados de saúde prestados a doentes com EM. Os registos de realização de RM também foram superiores nesta população, o que poderá sinalizar maior completude do processo clínico em períodos mais recentes. Observaram-se ainda diferenças na frequência de prescrição de terapêuticas modificadoras da doença, nomeadamente fumarato de dimetilo, o que espelha um aumento da diversidade de opções terapêuticas para a doença, ${ }^{14} \mathrm{e}$ das indicações especificas de prescrição.

Nas etapas de inserção e validação de dados vieram a constatar-se recorrentes lacunas na informação constante dos processos clínicos dos doentes. Estas falhas, em maior proporção para dados colhidos retrospetivamente, nomeadamente pontuação na escala EDSS ao diagnóstico e exames complementares de diagnóstico realizados (de que se destacam os registos de realização de RM somente em $33 \%$ dos doentes incluídos, encontrando-se os restantes omissos no processo clínico), refletem a heterogeneidade verificada na informação registada nos processos clínicos dos doentes com EM. Uma vez que o Registo depende de dados retrospetivos (inerência da sua fase de conceção e implementação), importa destacar a limitação que resulta dos dados omissos nos processos clínicos (com o potencial de sobre ou subvalorizar informação, fruto da sua coexistência com informação omissa para as mesmas variáveis em estudo). Cremos que esta limitação poderá ser ultrapassada através da definição de elementos obrigatórios a constar dos registos em processos de doentes com EM, que beneficiará a completude do Registo, nomeadamente na sua componente prospetiva.

Em termos de desafios na manutenção do Registo, estes verificaram-se também na recolha prospetiva de dados. Poderá argumentar-se que o número e a complexidade dos campos a preencher tenham tido um impacto negativo na facilidade e/ou frequência de completude, assim preconizando registos mais 'minimalistas' como forma de ultrapassar esta limitação. Importa porém reconhecer que a diminuição do número de dados e de variáveis poderá impactar a qualidade/utilidade do Registo.

Para além do corpo clínico do Serviço de Neurologia, a equipa de trabalho contou com a colaboração de outros profissionais na recolha de dados retrospetivos e prospetivos. Enquanto o corpo clínico esteve responsável 
pela apresentação do estudo aos doentes e pela recolha do formulário de consentimento informado, houve necessidade de envolver elementos adicionais na recolha de dados iniciais e na inserção de dados ao nível da plataforma (designadamente coordenadores de estudos clínicos e profissionais diferenciados em inserção de dados). Esta colaboração no decorrer do estudo permitiu libertar tempo aos clínicos para outras tarefas, nomeadamente a análise dos dados, e completar mais celeremente o Registo. Adicionalmente, a presença de elementos complementares à equipa médica potenciou a estandardização dos processos, aferida em equipa.

Deve, no entanto, ser discutido o custo e a eficiência da integração de elementos adicionais, cujo número dependerá do volume de informação a registar, do tempo previsto para a recolha de dados, e da população de doentes seguida ao nível do hospital. A redução do número de horas despendidas pelos clínicos com o concomitante aumento de horas afetas a outros profissionais permitiria a redução dos custos inerentes com potenciação da sustentabilidade do Registo. Também o desenvolvimento de registos mais 'minimalistas' (com redução do tempo de preenchimento) poderá ter um papel a este nível.

O cumprimento das boas práticas em investigação científica deve ser considerado em todos os passos de implementação de um registo médico, tendo também em atenção todas as questões éticas inerentes. O Registo desenvolvido pela nossa equipa assegura a anonimização dos dados recolhidos através da atribuição de um código numérico a cada doente e da limitação de acessos à plataforma de inserção de dados. Adicionalmente, foi garantido o armazenamento da informação em servidores seguros com acesso encriptado, cujos dados estão apenas acessíveis aos elementos da Keypoint - Consultoria Científica Lda., responsáveis pela condução do estudo.

Pelo exposto, consideramos que seria desejável estabelecer uma abordagem balanceada, através da definição de dados obrigatórios, da conferência regular de disparidades de dados por auditorias internas, da alocação da colheita e validação de dados a outros profissionais das equipas; e do auto-reporte de dados de caracterização e de seguimento pelos doentes.

Finalmente, consideramos que a motivação e participação dos profissionais poderá ser otimizada através do uso dos dados no Registo para apoio ao seguimento dos doentes de EM, pela inserção de novos elementos no interface, com foco nos cuidados personalizados; possibilidade de utilização do Registo para realização de avaliação estatística básica, através de módulo suplementar; inclusão, quando disponíveis, de coortes e dados epidemiológicos comparativos em relação à temática em estudo; utilização de aplicativos mais simples aquando da avaliação de doentes no serviço de urgência ou noutros locais, por exemplo em caso de surto; e presença de elementos adicionais que colaborem na recolha da informação e preenchimento do Registo.

\section{CONCLUSÃO}

Este trabalho descreve as etapas de criação e implementação de um registo de doentes num centro de esclerose múltipla em Portugal. A implementação do Registo consistiu na recolha retrospetiva e prospetiva de dados, tendo sido efetuada uma caracterização global (demográfica e clínica) dos doentes incluídos. No geral, os resultados evidenciaram que, apesar de se ter obtido um grande volume de informação da população de doentes com EM seguidos no HSM, a heterogeneidade na informação registada nos processos clínicos, o número e complexidade dos campos a preencher e, consequentemente, o tempo despendido pela equipa médica na inserção de dados, dificultaram a implementação e manutenção deste Registo. A definição de novas estratégias na implementação de Registo e o reforço da participação dos profissionais poderão permitir ultrapassar barreiras identificadas, alicerçando futuros registos a nível nacional.

\section{CONTRIBUTO DOS AUTORES}

JS: Contribuição substancial no desenho do estudo. Participação na recolha e interpretação de dados. Revisão crítica do conteúdo e aprovação da versão final do manuscrito.

JF: Participação na recolha e interpretação de dados. Aprovação da versão final do manuscrito.

AM: Contribuição substancial no desenho do estudo. Participação na análise e interpretação de dados. Participação na escrita e aprovação da versão final do manuscrito.

\section{PROTEÇÃO DE PESSOAS E ANIMAIS}

Os autores declaram que os procedimentos seguidos estavam de acordo com os regulamentos estabelecidos pelos responsáveis da Comissão de Investigação Clínica e Ética e de acordo com a Declaração de Helsínquia da Associação Médica Mundial actualizada em 2013.

\section{CONFIDENCIALIDADE DOS DADOS}

Os autores declaram ter seguido os protocolos do seu centro de trabalho acerca da publicação de dados.

\section{CONFLITOS DE INTERESSE}

Os autores declaram não ter conflitos de interesses relacionados com o presente trabalho.

\section{FONTES DE FINANCIAMENTO}

O projecto recebeu apoio técnico da Merck S.A., sob a forma de uma bolsa não restritiva.

\section{REFERÊNCIAS}

1. Richesson R, Vehik K. Patient registries: utility, validity and inference. Adv Exp Med Biol. 2010;686:87-104.
2. Jakobsen E, Green A, Oesterlind K, Rasmussen TR, lachina M, Palshof T. Nationwide quality improvement in lung cancer care: the role of the 
Danish Lung Cancer Group and Registry. J Thorac Oncol. 2013;8:123847.

3. Rankin EA. AJRR: becoming a National US Joint Registry. Orthopedics. 2013;36:175-6.

4. Delaunay C. Registries in orthopaedics. Orthop Traumatol Surg Res. 2015;101:S69-S75.

5. Ziemssen T, Kern R, Thomas K. Multiple sclerosis: clinical profiling and data collection as prerequisite for personalized medicine approach. BMC Neurol. 2016;16:1-10.

6. Lacaze P, Millis N, Fookes M, Zurynski Y, Jaffe A, Bellgard M, et al. Rare disease registries: a call to action. Intern Med J. 2017;47:1075-79.

7. Flachenecker P, Khil L, Bergmann S, Kowalewski M, Pascu I, PérezMiralles F, et al. Development and pilot phase of a European MS register. J Neurol. 2010;257:1620-7.

8. Butzkueven H, Chapman J, Cristiano E, Grand'Maison F, Hoffmann M, Izquerdo $\mathrm{G}$, et al. MSBase: an international, online registry and platform for collaborative outcomes research in multiple sclerosis. Mult Scler.
2006;12:769-74.

9. Fox R, Bacon T, Chamot E, Salter A, Cutter G, Kalina J, et al. Prevalence of multiple sclerosis symptoms across lifespan: data from the NARCOMS Registry. Neurodegener Dis Manag. 2015;5:3-10.

10. Howard J, Trevick S, Younger DS. Epidemiology of multiple sclerosis. Neurol Clin. 2016;34:919-39.

11. Sá MJ, Kobelt G, Berg J, Capsa D, Dalén J. New insights into the burden and costs of multiple sclerosis in Europe: results for Portugal. Mult Scler. 2017;23:S143-54.

12. Kobelt G, Thompson A, Berg J, Gannedahl M, Eriksson J. New insights into the burden and costs of multiple sclerosis in Europe. Mult Scler J. 2017;23:1123-36.

13. Confavreux $C$, Vukusic $S$. Natural history of multiple sclerosis: a unifying concept. Brain. 2006;129:606-16.

14. Cross AH, Naismith RT. Established and novel disease-modifying treatments in multiple sclerosis. J Intern Med. 2014;275:350-63. 